\title{
The Anterior Frontomedian Cortex and Evaluative Judgment: An fMRI Study
}

\author{
Stefan Zysset,* Oswald Huber,† Evelyn Ferstl,* and D. Y ves von Cramon* \\ *Max-Planck-Institute of Cognitive Neuroscience, D-04303 Leipzig, Germany; and †Department of Psychology, \\ University of Fribourg, Fribourg, Switzerland
}

Received J uly 9, 2001; published online February 7, 2002

This study investigated the neuronal basis of evaluative judgment. J udgments can be defined as the assessment of an external or internal stimulus on an internal scale and they are fundamental for decisionmaking and other cognitive processes. Evaluative judgments (I like George W. B ush: yes/no) are a special type of judgment, in which the internal scale is related to the person's value system (preferences, norms, aesthetic values, etc.). We used functional magnetic resonance imaging to examine brain activation during the performance of evaluative judgments as opposed to episodic and semantic memory retrieval. Evaluative judgment produced significant activation in the anterior frontomedian cortex (BA 10/9), the inferior precuneus (BA 23/31), and the left inferior prefrontal cortex (BA 45/47). The results show a functional dissociation between the activations in the anterior frontomedian cortex and in the inferior precuneus. The latter was mainly activated by episodic retrieval processes, supporting its function as a multimodal association area that integrates the different aspects of retrieved and newly presented information. In contrast, the anterior frontomedian cortex was mainly involved in evaluative judgments, supporting its role in self-referential processes and in the self-initiation of cognitive processes. 2002 EIsevier Science (USA)

\section{INTRODUCTION}

Evaluative judgments play an important role in everyday activities. They represent a central component of any choice process, because the decision-maker has to evaluate alternatives, attributes, the involved risk, etc. (cf. Hogarth, 1987). Evaluative judgments, however, are not restricted to decision-making. They are made in the context of personal preferences (I like this wine) or religious, aesthetic, social, or other values or in connection with attitudes. Despite the importance of evaluative judgments in our daily life, little is known about the neuronal implementation of the processes behind these judgments. The first goal of the present experiment was to measure cerebral activation when a person performs an evaluative judgment and to compare this activation with that in memory retrieval tasks.

A judgment generally can be defined as the assessment of an external or internal stimulus on an internal scale (e.g., subjective brightness of a light, pain, perceived sweetness of a liquid, etc.). Evaluative judgments (I like George W. Bush; yes/no) are a special type of judgment, in which the internal scale is related to the person's value system (preferences, norms, aesthetic values, etc.). Like memory retrieval tasks, evaluative judgments also depend on previously acquired knowledge and experienced events. They are closely linked to a person's value system, motives, internal goals, and objectives. In contrast to memory retrieval in general, many evaluative judgments are self-referential in that they refer to the subjects "narrative" self (Gallagher, 2000). The self involves personal identity and continuity across time, a long-term coherent whole of beliefs and attitudes (Vogeley et al., 1999), and includes a past and a future.

In order to isolate the evaluative process, we contrasted trials of evaluative judgment with memory retrieval trials. For memory retrieval tasks we differentiated between semantic (e.g., Berlin is the capital of Germany) and episodic (e.g., I have been to Berlin) retrieval (Tulving, 1972). Semantic memory retrieval refers to the recovery of encoded information from longterm memory (Graf et al., 1985). The retrieved information is matched against an externally given fact. In addition, an episodic memory retrieval condition was chosen as it represents the link between pure semantic retrieval and the evaluation of retrieved information. In episodic retrieval tasks, the retrieved information is unique to an individual and is tied to a specific context. Both semantic and episodic memory retrieval tasks depend on the previously encoded and retrieved information. Furthermore, questions can be designed in such a way that there exists an absolute objectively correct answer for a semantic memory retrieval task (New Orleans is the capital of Texas: yes/no). For epi- 


\section{TABLE 1}

Translations of Examples of Presented Stimuli

\begin{tabular}{ll}
\hline \multicolumn{1}{c}{ Task condition } & \multicolumn{1}{c}{ Example } \\
\hline Semantic memory retrieval & $\begin{array}{l}\text { Gerhard Schröder is the chancellor } \\
\text { of Germany. } \\
\text { Leipzig is the capital of Germany. } \\
\text { December 31st is New Year's Eve. }\end{array}$ \\
E pisodic memory retrieval & $\begin{array}{l}\text { I voted for Gerhard Schröder. } \\
\text { I was in Leipzig. }\end{array}$ \\
Evaluative judgment & $\begin{array}{l}\text { Gerhard Schröder is a good } \\
\text { chancellor. }\end{array}$ \\
& I like Leipzig. \\
I enjoy going to New Year's parties. \\
Baseline condition
\end{tabular}

Note In the actual experiment, the sentences were presented in the German language. 60 items for each of these conditions were generated and presented in random order. Subjects responded by pressing a button based on whether or not they agreed with the statement.

sodic retrieval tasks, an objectively correct answer exists and may be different for different subjects (e.g., You might have been in New Orleans but I may have not been there). For the evaluative judgment task, stored information needs to be retrieved as well. But over and above retrieval and matching, the statement has to be evaluated internally. For evaluative tasks there is no objectively correct solution (e.g., Wine tastes better than beer: yes/no).

In our study, a sentence is presented visually to the subject (see Table 1). She/he has to decide whether she/he agrees with this statement. The cerebral activation was measured with $\mathrm{FMRI}$ using the BOLD contrast (Ogawa \&t al., 1992; Belliveau et al., 1991) while the subjects performed judgment tasks and memory retrieval tasks. By contrasting the hemodynamic re sponse of evaluative trials with trials of semantic and episodic retrieval, we should be able to locate regions involved in these evaluative processes.

\section{METHOD}

\section{Subjects}

We obtained written consent from all 13 subjects (mean age 26 years; 5 female, 8 male) prior to the scanning session. All subjects had normal or correctedto-normal vision and were native German speakers. Subjects were instructed about the task prior to the actual experimental session.

\section{Psychological Procedures}

We used knowledge items which allowed for the formulation of questions for the evaluative judgment task and the two types of memory retrieval task (for exam- ples see Table 1). An additional baseline condition ("Press left button") was used in order to control for perceptual factors, response preparation, and motor execution. Further, null events (empty trials) were introduced to improve the statistical evaluation of the data (Miezin et al., 2000).

Altogether, there were five conditions: evaluative judgment, semantic memory retrieval, episodic memory retrieval, baseline condition, and null events (a sixth experimental condition is not reported here). Sixty items were generated for each of these conditions, for a total number of 360 trials, of which 300 are reported here. Half of the trials were positive assertions and the other half negative. The semantic retrieval tasks were pretested so that at least $90 \%$ of the responses were correct. The episodic and evaluative trials were pretested too, for an equal distribution of Yes and No responses. The conditions were presented in a randomized order. Stimulus sentences were displayed by a LCD projector on a back-projection screen mounted in the bore of the magnet behind the participant's head. Participants viewed the screen wearing mirror glasses. Depending on the length of the sentences, the stimuli were displayed across several lines. The stimulus sentences appeared on the screen for $2 \mathrm{~s}$, after which they disappeared, independent of whether a response had been given. The pretests showed that $2 \mathrm{~s}$ were sufficient for reading and understanding the sentences. Subjects were given $4 \mathrm{~s}$ after stimulus onset to respond by pressing with the right index finger (Yes) or right middle finger (No). The screen remained blank between trials. Stimuli were presented every $6 \mathrm{~s}$ on average. In order to increase the temporal resolution, the trials were presented with variable onset delays of $0,400,800,1200$, or $1600 \mathrm{~ms}$. This produced an oversampling of the actual image acquisition time of 2000 ms by a factor of 5 (Miezin et al., 2000).

\section{MRI Scanning Procedure}

The experiment was carried out on a 3-T scanner (Medspec 30/100; Bruker, Ettlingen). Sixteen axial slices (19.2-cm FOV, 64 by 64 matrix, 5-mm thickness, 1-mm spacing), parallel to the AC-PC plane and covering the whole brain, were acquired using a single-shot, gradient-recalled EPI sequence (TR 2000 ms, TE 30 $\mathrm{ms}, 90^{\circ}$ flip angle). Three functional runs with 363 time points each were run, with each time point sampling over the 16 slices. Prior to the functional runs, 16 anatomical T1-weighted MDEFT (Ugurbil et al., 1993; Norris, 2000) images (data matrix $256 \times 256$, TR $1.3 \mathrm{~s}$, TE $10 \mathrm{~ms}$ ) and 16 T1-weighted EPI images with the same parameters as the functional data were acquired.

\section{fMRI Data Analysis}

The fMRI data were processed on a SGI Origin 2000 with in-house software LIPSIA (Lohmann et al., 2001). 


\section{TABLE 2}

Talairach Coordinates (for the Evaluative vs Semantic Condition) and Maximum Z Value of the Local Maxima (Volume of the Activated Region) for Three Different Contrasts: E pisodic Memory Retrieval vs Semantic Memory Retrieval, Evaluative J udgment vs Semantic Memory Retrieval, and Evaluative J udgment vs E pisodic Memory Retrieval

\begin{tabular}{|c|c|c|c|c|}
\hline \multirow[b]{2}{*}{ Area (Brodmann area) } & \multirow[b]{2}{*}{ Talairach coordinates } & \multicolumn{3}{|c|}{ Statistical effect } \\
\hline & & E pisodic vs semantic & Evaluative vs semantic & Evaluative vs episodic \\
\hline Frontomedian cortex (10m/9m) & -65513 & $4.36(2,245)$ & $4.69(5,761)$ & 4.37 (432) \\
\hline Precuneus/PCC (23/31) & $-10-5136$ & $5.20(10,711)$ & $4.17(1,700)$ & $-5.93(11,944)$ \\
\hline L. inferior frontal gyrus (45/47) & $-4216-4$ & & $4.96(1,914)$ & $4.56(2,336)$ \\
\hline L. superior frontal sulcus $(6 / 8)$ & 291746 & & & $-4.80(1,337)$ \\
\hline R. superior frontal sulcus $(6 / 8)$ & -251548 & & & $-5.06(1,437)$ \\
\hline R. middle frontal gyrus (8) & 472925 & & $-4.20(1,695)$ & $-4.33(557)$ \\
\hline L. inferior frontal gyrus (47) & -382613 & $-4.88(817)$ & & \\
\hline L. frontal eye field (6) & $-39-1447$ & & $-4.43(1,315)$ & \\
\hline L. inferior precentral gyrus & -49126 & $-4.53(1,134)$ & $-4.21(867)$ & \\
\hline R. inferior precentral gyrus & 43329 & $-4.82(1,879)$ & $-3.91(773)$ & \\
\hline L. hippocampus & $-22-20-12$ & $4.11(348)$ & & \\
\hline L. intraparietal sulcus (7) & $-33-5748$ & $-4.60(2,762)$ & $-5.00(11,325)$ & $-5.32(4,226)$ \\
\hline R. intraparietal sulcus (7) & $30-7635$ & $-4.81(4,834)$ & $-5.50(12,388)$ & $-5.09(5,452)$ \\
\hline L. superior occipital sulcus (39) & $-54-6327$ & $4.15(1,273)$ & 4.04 (384) & \\
\hline R. superior occipital sulcus (39) & $39-7811$ & $-4.15(585)$ & & \\
\hline L. fusiform gyrus (37) & $-47-683$ & $-5.47(6,185)$ & $-4.91(4,352)$ & \\
\hline R. fusiform gyrus (37) & $48-34-8$ & $-4.12(567)$ & $-4.91(2,032)$ & \\
\hline
\end{tabular}

Note Only activations with a $Z$ value $>3.1$ and with a volume greater than $324 \mathrm{~mm}^{3}$ ( 6 voxels) are reported here. Negative $Z$ values represent a higher activation under the semantic (episodic resp) condition compared to the episodic or evaluative condition.

This software package contains tools for preprocessing, registration, statistical evaluation, and visualization of fMRI data.

Prior to the reconstruction of the functional data, the three corresponding runs were concatenated into a single run. Functional data were corrected for motion using a matching metric based on linear correlation. To correct for the temporal offset between the slices acquired in one scan, a sinc-interpolation based on the Nyquist-Shannon Theorem was applied. A temporal high-pass filter with a cutoff frequency of $1 / 72 \mathrm{~Hz}$ was used for baseline correction of the signal and a spatial Gaussian filter with $5.65 \mathrm{~mm}$ FWHM was applied. The increased autocorrelation due to filtering was taken into account during statistical evaluation.

To align the functional data slices onto a 3D stereotactic coordinate reference system, a rigid linear registration with 6 degrees of freedom ( 3 rotational, 3 translational) was performed. The rotational and translational parameters were acquired on the basis of the MDEFT and EPI-T1 slices to achieve an optimal match between these slices and the individual 3D reference data set. This 3D reference data set was acquired for each subject during a previous scanning session. The 3D MDEFT (Ugurbil et al., 1993; Lee ef al., 1995) volume data set (128 sagittal slices, $1.5-\mathrm{mm}$ thickness, FOV $25.0 \times 25.0 \times 19.2 \mathrm{~cm}$, data matrix of 256 by 256 ) was standardized to the Talairach stereotactic space (Talairach and Tournoux, 1988). The same rotational and translational parameters were normal- ized, i.e., transformed by linear scaling to a standard size. Then, the resulting parameters were used to transform the functional slices using trilinear interpolation, so that the resulting functional slices were aligned with the stereotactic coordinate system. This linear normalization process was improved by a subsequent processing step that performed an additional nonlinear normalization (Thirion, 1998).

The statistical evaluation was based on a leastsquares estimation using the general linear model for serially autocorrelated observations (Friston, 1994; Friston et al., 1995; Worsley and Friston, 1995; Aguirre et al., 1997; Zarahn et al., 1997). The design matrix was generated utilizing a synthetic hemodynamic response function (Friston et al., 1998) and a response delay of $6 \mathrm{~s}$. In order to compensate for the time it takes to read the presented sentence, the onset time point of each event was set to half the length of the individual reaction time. In this way, differences in reading duration between subjects and between different conditions were leveled out. The model equation, including the observation data, the design matrix, and the error term, was convolved with a Gaussian kernel with a dispersion of $4 \mathrm{~s} \mathrm{FWHM.} \mathrm{Within} \mathrm{this} \mathrm{model,} \mathrm{the} \mathrm{tem-}$ poral autocorrelation and the effective degrees of freedom were estimated ( $\mathrm{df}=461$; Worsley and Friston, 1995; Seber, 1977). In the following, contrast maps, i.e., estimates of the raw-score differences of the $\beta$ coefficients between specified conditions, were generated for each subject. As the individual functional data sets 
A

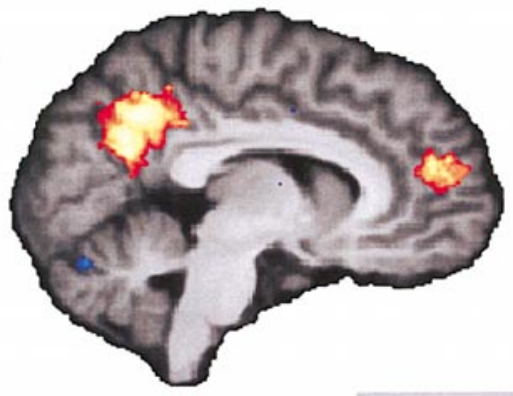

$3.7=5.7$
1

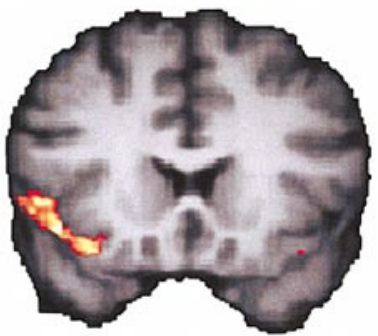

A Fronto-median cortex

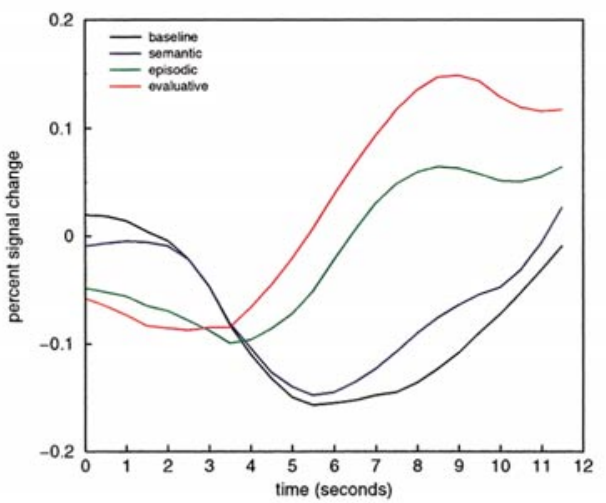

C Left inferior frontal gyrus

2



C

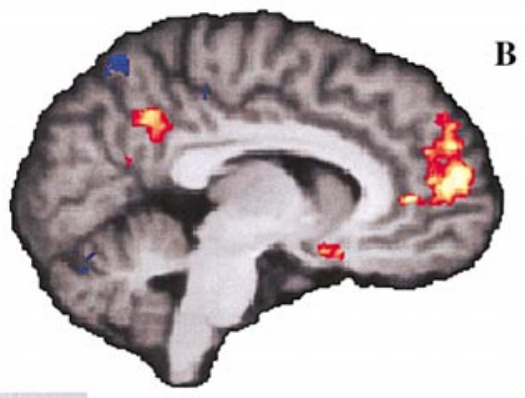

B

5.4

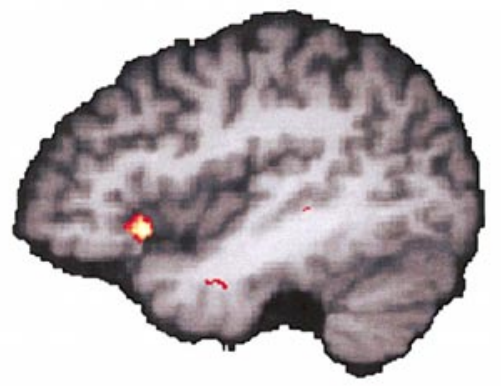

B Precuneus

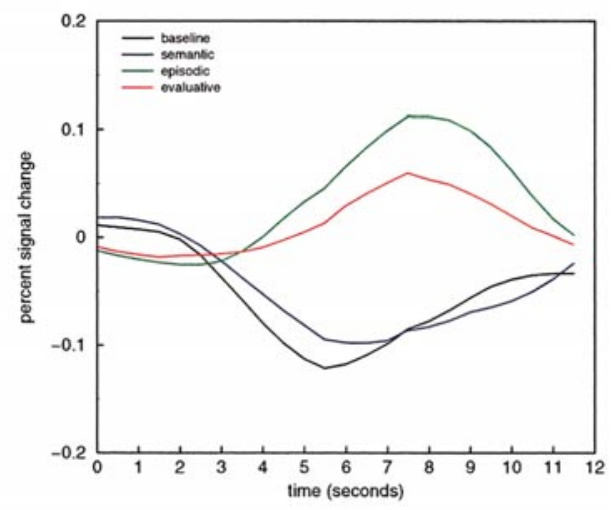

D Visual cortex

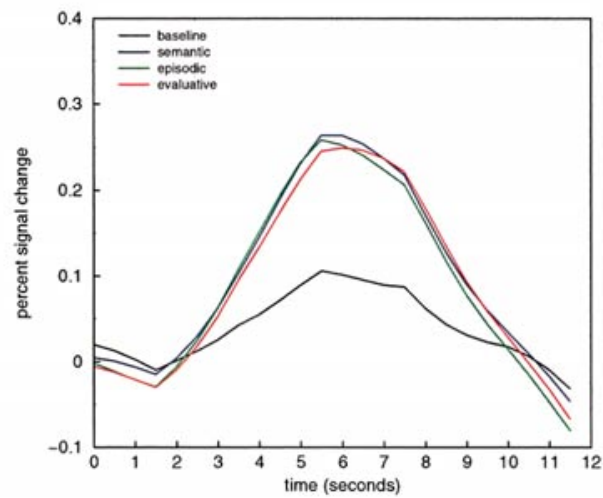

FIG. 1. Activation maps averaged over all 13 subjects ( $Z$ thresholded at $Z=3.1$ ) mapped onto the mean brain of all subjects. (A) View of the medial surface $(x=-7)$ for the contrast of episodic vs semantic; $(B)$ View of the medial surface $(x=-7)$ for the contrast evaluative vs semantic, (C) Coronar $(y=16)$ and sagittal $(x=-42)$ view of the activation in the inferior frontal gyrus in the evaluative vs semantic contrast $(x=-48)$. Red/yellow labels indicate positive $Z$ values, whereas blue labels indicate negative $Z$ values.

FIG. 2. Averaged time lines of regions of interest. The voxel with the highest activation in the average $Z$ map and its 26 neighboring voxels were chosen. The signal was averaged over all 27 voxels and all 13 subjects. The signal was averaged for the onset of the presentation of the stimulus. 
were all aligned to the same stereotactic reference space, a group analysis was subsequently performed. A one-sample t test of contrast maps across subjects was computed to indicate whether observed differences be tween conditions were significantly different from zero (Holmes and Friston, 1998). Subsequently, t values were transformed into $Z$ scores. To protect against false positive activations, only regions with a $Z$ score greater than 3.1 ( $P<0.001$ uncorrected) and with a volume greater than $324 \mathrm{~mm}^{3}$ (6 voxels) were considered (Braver et al., 2001).

\section{RESULTS}

\section{Behavioral Results}

For the episodic and the evaluative conditions it was not possible to differentiate between correct and false responses, so that all responses were considered. The reaction times (RT) were first averaged for each subject and each condition, independent of the response given, and second averaged over all subjects. For the baseline condition, mean RT was $1078 \mathrm{~ms}$ (SE $=76 \mathrm{~ms}$ ). For the three task conditions, the resulting mean RT was 2035 $\mathrm{ms}$ (SE $=117 \mathrm{~ms}$ ) for the semantic condition, $1985 \mathrm{~ms}$ (SE $=125 \mathrm{~ms}$ ) for the episodic condition, and $2111 \mathrm{~ms}$ (SE $=120 \mathrm{~ms}$ ) for the evaluative condition. An ANOVA over the three task conditions (the baseline condition was not included) showed that these means did not differ significantly from each other $(F(2,36)=0.261$; $P>0.1$ ).

Errors made in the semantic memory retrieval condition amounted to 5.6\%. Of the episodic and the evaluative questions, 48.5 and $51.5 \%$, respectively, were answered Yes. The fact that for both conditions, the episodic as well as the evaluative, about $50 \%$ Yes and No responses were given shows that the questions were well matched and no response tendencies were produced.

\section{Imaging Results}

First, we contrasted the semantic memory retrieval condition, the episodic memory retrieval condition, and the evaluative judgment condition against each other in order to investigate the neuronal network of evaluative judgment. The results are reported in Table 2 and in Fig. 1.

Further, the time courses of the underlying BOLD signal were extracted for regions of interest (ROI). We chose the three main activations, which were the frontomedian cortex, the precuneus, the left fronto-opercular cortex, and as a reference point the primary visual cortex. Within these regions, the voxel with the highest $Z$ value was chosen. The signal intensities of this voxel and its 26 neighbors were averaged for each of the four relevant conditions and over all 13 subjects. The time

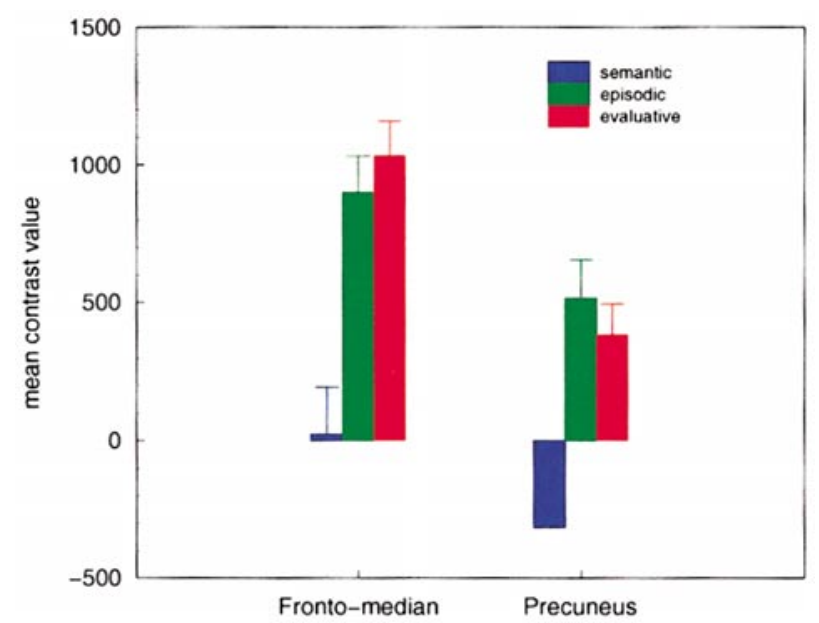

FIG. 3. Mean contrast values of regions of interest. The contrast values of the semantic, episodic, and evaluative condition (contrasted against the baseline condition) were averaged over all subjects and the regions of interest. The ANOVA showed significant effects for the frontomedian cortex (left; $\mathrm{P}<0.001$ ) and the precuneus (right; $\mathrm{P}<$ $0.001)$, but not for the inferior frontal gyrus and the visual cortex ( $P>0.05$, not shown in the graph).

course of the null events was subtracted from the averaged time courses. The resulting graphs are shown in Fig. 2.

When contrasting the episodic or evaluative conditions with the semantic condition, there were major activations in the anterior frontomedian cortex (BA $10 \mathrm{~m} / 9 \mathrm{~m}$ ) and in the inferior precuneus (BA 23/31; see Figs. $1 A$ and $1 B$ ) and a minor activation in the left superior occipital sulcus. The anterior frontomedian cortex was activated only by the two conditions which involved self-referential processes and not by the mere retrieval of semantic information (see Fig. 2A). It was mostly activated by the evaluative judgment condition and less by the episodic retrieval condition. A ROI analysis (Bosch, 2000) of the contrast values showed a significant difference between the episodic and the evaluative condition (one-sided t test; $t=5.93, \mathrm{P}<$ 0.05; see Fig. 3), which could also be seen when contrasting the evaluative against the episodic condition.

The inferior tip of the precuneus was activated mainly by the episodic task, less by the evaluative task (see Fig. 2B). A signal loss occurred during the semantic task. The ROI analysis showed a significant difference between the episodic and the semantic condition (one-sided t test; $\mathrm{t}=5.46, \mathrm{P}<0.01$; see Fig. 3). In addition, the evaluative condition showed a stronger activation than the semantic condition in the left inferior frontal gyrus (BA 45/47), extending into the frontoopercular cortex (see Fig. 1C). The time lines showed that the left fronto-opercular cortex was engaged in all three conditions but most strongly in the evaluative judgment condition and to a lesser extent in the semantic and episodic condition (see Fig. 2C). For this 
region and the primary visual cortex, the contrast values did not differ significantly between the three conditions $(F(2,36)=2.29, P=0.115 ; F(2,36)=0.62, P=$ 0.554, respectively). Further, the episodic condition produced stronger activations in the left hippocampus.

The semantic condition produced stronger activation than the episodic and evaluative conditions in the inferior precentral gyrus (bilateral), the fusiform gyrus (bilateral), and the intraparietal sulcus (bilateral; see Table 2). The semantic condition produced stronger activations than the episodic condition in the left inferior frontal gyrus and right superior occipital sulcus. The semantic condition produced stronger activations than the evaluative condition in the left frontal eye field and the right middle frontal gyrus.

When contrasting the episodic with the evaluative condition, stronger activations for the evaluative condition were found only in the left fronto-opercular cortex and the frontomedian cortex (see Table 2). The episodic condition elicited stronger activation than the evaluative condition in the left and right superior frontal sulcus, the right middle frontal gyrus, the inferior precuneus, and the left and right intraparietal sulcus.

Figure 2D shows the referential time lines in the visual cortex. No differences between the three task conditions can be seen. The baseline condition, in which only a standard sentence was read, shows a reduced signal increase.

\section{DISCUSSION}

For both evaluative judgment and episodic memory retrieval strong activations were found in the anterior frontomedian cortex (BA 10/9), in the inferior part of the precuneus, and additionally for the evaluative task, in the left inferior frontal cortex (BA 45/47). In monkeys, the frontomedian cortex and the inferior precuneus are interconnected through dense corticocortical connections (Barbas, 1992; Pandya and Yeterian, 1996) and it is assumed that the interconnecting fibers travel along the medial surface of the parietal and frontal lobes dorsal to the cingulate gyrus (Petrides and Pandya, 1984). The findings of our study demonstrate the functional interdependence of the anterior frontomedian and the median parietal cortex. The extent of the activation in each region appears to be dependent on the specific task set. The anterior frontomedian cortex was activated mostly by the two conditions that required self-referential processing of the information in contrast with the mere retrieval of semantic information. Activation of the anterior frontomedian cortex was more pronounced in the evaluative condition compared to the episodic condition, whereas for the inferior precuneus activation the reverse was true.

\section{Inferior Frontal Gyrus}

The left inferior frontal gyrus was engaged in all three task conditions but not in the baseline condition. The activation was stronger in the evaluative judgment condition than in the semantic and episodic conditions. It has been argued that the inferior frontal gyrus is involved in the selection of aspects of available information (Thompson-Schill et al., 1997). Whereas in the semantic and episodic condition the number of competing alternatives is limited to 2 (e.g., have been there vs have not been there), in the evaluative condition the alternatives are not that clearly defined and responses are more ambiguous. There are also only two possible answers (Yes or No), but the discrimination is more gradual. The statement "I like Leipzig" is evaluated on a scale with more than two levels, probably a continuum from "very much" to "not at all," rather than assigned a simple "Yes" or "No" response. Furthermore, it seems plausible that in the evaluative condition, connections between a multitude of retrieved items are activated, thus leading to a greater semantic processing load. In other words, we argue that more information processing is necessary in the evaluative condition, leading to a stronger hemodynamic response.

\section{Medial Prefrontal Region}

Anterior frontomedian activations comparable to those in the present study have been reported in studies investigating "theory of mind" (Premack and Wooldruff, 1978; Fletcher et al., 1995b; Goel et al., 1995). Theory of mind refers to the ability to attribute independent mental states to the self and others in order to explain and predict behavior (Gallagher et al., 2000). The present study does not involve the explanation or prediction of the mental states of the self and others in order to explain behavior. Because of this, theory of mind does not appear a necessary explanation for the presented activations. Rather, evaluative judgment demands the person's own attitudes and preferences. One can argue, however, that theory of mind is based on autobiographical experiences and self-referential processes that are projected onto another person's mind (Vogeley et al., 1999). Gusnard and colleagues (2001) argue that the anterior frontomedian cortex is essential for self-referential mental activity (see also Raichle et al., 2001). Activity in the frontomedian cortex is increased when attention is directed specifically toward self-referential or introspectively oriented mental activity. Maguire and Mummery (Maguire et al., 1999) found in their study anterior frontomedian activation for the verification of memory in reference to oneself. Other functional imaging studies reported anterior frontomedian activation for self-initiated thoughts (McGuire et al., 1996a), processing of information about intentions (Castelli et al., 2000) and intended 
speech (McGuire et al., 1996b), as well as subjective emotional responses (Lane et al., 1997; for an overview see Gusnard et al., 2001; Castelli et al., 2000). In conclusion, it appears that the anterior frontomedian cortex plays an important role in self-referential processes.

Evaluative judgment can also be seen as a self-referential process, and by this, the present anterior frontomedian activation fits well into the published findings. But it has also been shown that it occurred with tasks which were not explicitly self-referential (Ferstl and von Cramon, 2001; Goel et al., 1997). In the study of Ferstl and von Cramon, which investigated the establishment of coherence between sentence pairs, an anterior frontomedian activation was found, comparable to that in the present study. It was argued that the anterior frontomedian cortex is involved in the selfinitiation of a cognitive process in the context of tasks that require the active utilization of the individual's background knowledge. In particular, the anterior frontomedian cortex is implicated in the generation of ideas and plans for situations in which the solution is not inherent in the presented situation. In the semantic and episodic condition, the different retrieved items "match" or "mismatch" within the memory network and the response is obvious. The semantic and episodic memory retrieval task corresponds to veridical decision-making, based on the identification of the correct response, which is inherent in the presented assertion (Goldberg and Podell, 1999). On the other hand, an evaluative judgment task can be seen as adaptive decision-making, guided by the subject's priorities. The evaluative judgment task required the retrieval of semantic and episodic memory traces, but additionally some sort of mediator process (Burgess and Shallice, 1996) will be necessary. This process controls strategic and problem-solving operations, which are distinct from memory retrieval processes and represent a complex set of problem-solving routines. This metalevel process modulates the interaction among systems (J ohnson-Laird, 1983; Kuhl, 1994), as opposed to within-subsystem conflicts, which may be resolved automatically. By this, evaluative judgments can be seen as a volitional process (Nuttin, 1987; Kuhl, 1981, 1994) reflecting the realization of intentions and implementation of a metacontrol level (Kuhl and Goschke, 1994). The underlying process must be introspectively oriented, bridging the gap between the intention of an action and its realization. It appears that the anterior frontomedian cortex supports this metacontrol process, on which self-referential, theory of mind, and other processes are based.

Furthermore, one could argue that anterior frontomedian activation is elicited by emotional arousal induced by the thought of a specific situation or fact. Emotional perception, judgment, and behavior can proceed automatically, outside of conscious awareness
(Lazarus, 1991; LeDoux, 1996; Ohman and Soares, 1993). In a PET study conducted by Lane et al. (1998), activations in the anterior cingulate cortex (ACC) were associated with emotional arousal. However, the same emotional responses should be aroused for the semantic, episodic, and evaluative condition as the same situation or fact is retrieved. Therefore, no differential activation is expected for contrasting the three experimental conditions against each other. And indeed, the ACC was not differentially activated by the three conditions, supporting this notion. The anterior frontomedian activation seems specific to a consciously controlled prosses, not to an automatic emotional arousal process. Otherwise, semantic retrieval should have activated this region as well.

\section{Precuneal Region}

The inferior part of the precuneus was mainly activated by the episodic retrieval task and less by the evaluative task. A signal loss occurred during the semantic retrieval task. In the aforementioned study by Ferstl and von Cramon (2001) activations in the anterior frontomedian cortex and the inferior precuneus co-occured. The authors argued that the inferior precuneus is necessary for the encoding of a newly formed situation model representation. Further studies reported that the inferior precuneus plays a role in forming a mental model by integrating the current input with the background knowledge or the previously established situation model (Maguire et al., 1999; Fletcher et al., 1995a). The precuneus has also been identified as a multimodal association area, especially in episodic memory retrieval (Krause et al., 1999). The demand on the precuneus is higher in episodic and evaluative tasks than in semantic tasks, as these conditions depend more on the interconnections between retrieved information and prior knowledge. Further, in the episodic condition, demands on the precuneus are greater than in the evaluative condition, as the retrieved information about a past event must be integrated into an already existing situation model, whereas in the evaluative condition, no match against an existing situation model is necessary.

\section{CONCLUSION}

The process of evaluative judgment appears to be implemented in a neuronal network containing the anterior frontomedian cortex, the inferior precuneus, and the left inferior frontal gyrus. The inferior frontal gyrus is involved in evaluative judgment for the selection of available information among competing alternatives. A clear dissociation between the underlying processes of the inferior precuneus and the anterior frontomedian cortex could be made, with both regions being strongly interdependent. Evaluative judgment, 
which can be referred to as a metacontrol process, appears to be implemented in the anterior frontomedian cortex. The inferior precuneus serves to update the situation model, a region which is strongly needed for episodic memory retrieval.

\section{ACKNOWLEDGMENTS}

The authors thank Andrea Samson and Marc Bär for conceptualizing and testing the stimuli and Anke Mempel, Mandy Naumann, and Katrin Wiesner for conducting the fMRI experiment.

\section{REFERENCES}

Aguirre, G. K., Zarahn, E., and d'Esposito, M. 1997. Empirical analyses of BOLD fMRI statistics. II. Spatially smoothed data collected under null-hypothesis and experimental conditions. Neurol mage 5: 199-212.

Barbas, H. 1992. Architecture and cortical connections of the prefrontal cortex in the rhesus monkey. Adv. Neurol. 57: 91-115.

Belliveau, J. W., Kennedy, D. N., McKinstry, R. C., Buchbinder, B. R., Weisskoff, R. M., Cohen, M. S., Vevea, J . M., Brady, T. J ., and Rosen, B. R. 1991. Functional mapping of the human visual cortex by magnetic resonance imaging. Science 254: 716-719.

Bosch, V. 2000. Statistical analysis of multi-subject fMRI data: The assessment of focal activations. J . Magn. Reson. Imaging 11: 6164.

Braver, T. S., Barch, D. M., Kelley, W. M., Buckner, R. L., Cohen, N. J ., Miezin, F. M., Snyder, A. Z., Ollinger, J . M., Akbudak, E., Conturo, T. E., and Petersen, S. E. 2001. Direct comparison of prefrontal cortex regions engaged in working memory and longterm memory tasks. Neurol mage 14: 48-59.

Burgess, P. W., and Shallice, T. 1996. Confabulation and the control of recollection. Memory 4: 359-411.

Castelli, F., Happe, F., Frith, U., and Frith, C. 2000. Movement and mind: A functional imaging study of perception and interpretation of complex intentional movement patterns. Neurol mage 12: 314325.

Ferstl, E. C., and von Cramon, D. Y. 2001. The role of coherence and cohesion in text comprehension: An event-related fMRI study. Cognit. Brain Res. 11: 325-340.

Fletcher, P. C., Frith, C. D., Grasby, P. M., Shallice, T., Frackowiak, R. S. J ., and Dolan, R. J . 1995a. Brain systems for encoding and retrieval of auditory-verbal memory: An in vivo study in humans. Brain 118: 401- 416.

Fletcher, P. C., Happe, F., Frith, U., Baker, S. C., Dolan, R. J ., Frackowiak, R., and Frith, C. 1995b. Other minds in the brain: A functional imaging study of "theory of mind' in story comprehension. Cognition 57: 109-128.

Friston, K. J . 1994. Statistical parametric maps in functional imaging: A general linear approach. Hum. Brain Mapping 2: 189-210.

Friston, K. J ., Fletcher, P., J osephs, O., Holmes, A., Rugg, M. D., and Turner, R. 1998. Event-related fMRI: Characterizing differential responses. Neurol mage 7: 30-40.

Friston, K. J ., Holmes, A. P., Poline, J .-B., Grasby, B. J ., Williams, C. R., Frackowiak, R. S. J ., and Turner, R. 1995. Analysis of fMRI time-series revisited. Neurol mage 2: 45-53.

Gallagher, H. L., Happe, F., Brunswick, N., Fletcher, P., Frith, U., and Frith, C. D. 2000. Reading the mind in cartoons and stories: An fMRI study of 'theory of mind' in verbal and nonverbal tasks. Neuropsychologia 38: 11-21.

Gallagher, S. 2000. Philosophical conceptions of the self: Implications for cognitive science. Trends Cognit. Sci. 4: 127-136.
Goel, V., Gold, B., Kapur, S., and Houle, S. 1997. The seats of reason? An imaging study of deductive and inductive reasoning. NeuroReport 8: 1305-1310.

Goel, V., Grafman, J ., Sadato, N., and Hallett, M. 1995. Modeling other minds. NeuroReport 6: 1741-1746.

Goldberg, E., and Podell, K. 1999. Adaptive versus veridical decision making and the frontal lobes. Consciousness Cognit. 8: 364-377.

Graf, P., Shimamura, A. P., and Squire, L. R. 1985. Priming across modalities and priming across category levels: Extending the domain of preserved function in amnesia. J . Exp. Psychol. Learning Memory Cognit. 11: 386-396.

Gusnard, D. A., Akbudak, E., Shulman, G. L., and Raichle, M. E. 2001. Medial prefrontal cortex and self-referential mental activity: Relation to a default mode of brain function. Proc. Nat. Acad. Sci. USA 98: 4259-4264.

Hogarth, R. M. 1987. J udgement and Choice. Chichester, New York. Holmes, A. P., and Friston, K. J . 1998. Generalisability, random effects and population inference. Neurol mage 7(Suppl.): 754.

J ohnson-Laird, P. N. 1983. Mental Models. Toward a Cognitive Science of Language, Inference, and Consciousness. Cambridge Univ. Press, Cambridge, UK.

Krause, B. J ., Schmidt, D., Mottaghy, F. M., Taylor, J ., Halsband, U., Herzog, H., Tellmann, L., and Müller-Gärtner, H.-W. 1999. Episodic retrieval activates the precuneus irrespective of the imagery content of word pair associates: A PET-study. Brain 122: 255-263.

Kuhl, J . 1981. Motivational and functional hel plessness: The moderating effect of state versus action orientation. J . Pers. Soc. Psychol. 40: 155-170.

Kuhl, J. 1994. Volitional aspects of achievement motivation and learned helplessness: Toward a comprehensive theory of actioncontrol. In Progress in Experimental Personality Research (B. A. Maher, Ed.), Band 13, pp. 99-171. Academic Press, New York.

Kuhl, J ., and Goschke, T. 1994. A theory of action control: Mental subsystems, modes of control, and volitional conflict-resolution strategies. In Volition and Personality: Action versus State Orientation (J. Kuhl and J. Beckmann, Eds.), pp. 93-124. Hogrefe \& Huber, Seattle:

Lane, R. D., Fink, G. R., Chau, P. M. L., and Dolan, R. J . 1997. Neural activation during selective attention to subjective emotional responses. NeuroReport 8: 3969-3972.

Lane, R. D., Reiman, E. M., Axelrod, B., Yun, L.-S., Holmes, A., and Schwartz, G. E. 1998. Neuronal correlates of levels of emotional awareness: Evidence of an interaction between emotion and attention in the anterior cingulate cortex. J . Cognit. Neurosci. 10: 525535.

Lazarus, R. S. 1991. Emotion and Adaptation. Oxford Univ. Press, London.

LeDoux, J . E. 1996. The Emotional Brain. Simon \& Schuster, New York.

Lee, J .-H., Garwood, M., Menon, R., Adriany, G., Andersen, P., Truwit, C. L., and Ugurbil, K. 1995. High contrast and fast threedimensional magnetic resonance imaging at high fields. Magn. Reson. Med. 34: 308.

Lohmann, G., Müller, K., Bosch, V., Mentzel, H., Hessler, S., Chen, L., and Zysset, S., and von Cramon, D. Y. 2001. Lipsia-A new software system for the evaluation of functional magnetic resonance images of the human brain. Comput. Med. I maging Graph., in press.

Maguire, E. A., Frith, C. D., and Morris, R. G. M. 1999. The functional neuroanatomy of comprehension and memory: The importance of prior knowledge. Brain 122: 1839-1850.

McGuire, P. K. Paulesu, E., Frackowiak, R. S. J ., and Frith, C. D. 1996a. Brain activity during stimulus independent thought. NeuroReport 7: 2095-2099. 
McGuire, P. K., Silbersweig, D. A., and Frith, C. D. 1996b. Functional neuroanatomy of verbal self-monitoring. Brain 119: 907-917.

Miezin, F. M., Maccotta, L., Ollinger, J. M., Petersen, S. E., and Buckner, R. L. 2000. Characterizing the hemodynamic response: Effects of presentation rate, sampling procedure, and the possibility of ordering brain activity based on relative timing. Neurol mage 11: $735-759$.

Norris, D. G. 2000. Reduced power multi-slice MDEFT imaging. J . Magn. Reson. Imaging 11: 445- 451.

Nuttin, J . R. 1987. The respective roles of cognition and motivation in behavioral dynamics, intention, and volition. In Motivation, Intention and Volition ( $F$. Halisch and J. Kuhl, Eds.), Chap. 20, (pp. 309-320). Springer-Verlag, Berlin.

Ogawa, S., Tank, D. W., Menon, R. Ellermann, J. M., Kim, S. G., Merkle, H., and U gurbil, K. 1992. Intrinsic signal changes accompanying sensory stimulation: Functional brain mapping with magnetic resonsance imaging. Proc. Natl. Acad. Sci. USA 89: 59515955.

Ohman, A., and Soares, J . J . E. 1993. Unconscious anxiety: Phobic responses to masked stimuli. J . Abnorm. Psychol. 103: 231-240.

Pandya, D. N., and Yeterian, E. H. 1996. Comparison of prefrontal architecture and connections. Philos. Trans. R. Soc. London 351: 1423-132.

Petrides, M., and Pandya, D. N. 1984. Projections to the frontal cortex from the posterior parietal region in the rhesus monkey. J . Comp. Neurol. 228: 105-116.

Premack, D., and Wooldruff, G. 1978. Does the chimapanzee have a theory of mind? Behav. Brain Sci. 4: 515-526.
Raichle, M. E., MacLeod, A. M., Snyder, A. Z., Powers, W. J ., Gusnard, D. A., and Shulman, G. L. 2001. A default mode of brain function. Proc. Natl. Acad. Sci. USA 98: 676- 682.

Seber, G. A. F. 1977. Linear Regression Analysis. Wiley, New York. Talairach, P., and Tournoux, J . 1988. A Stereotactic Coplanar Atlas of the Human Brain. Thieme, Stuttgart.

Thirion, J. P. 1998. Image matching as a diffusion process: An analogy with Maxwell's demons. Med. Image Anal. 2: 243-260.

Thompson-Schill, S. L., D’E sposito, M., Aguirre, G., and Farah, M. J . 1997. Role of left inferior prefrontal cortex in retrieval of semantic knowledge: A reevaluation. Proc. Natl. Acad. Sci. USA 94: 1479214797.

Tulving, E. 1972. E pisodic and semantic memory. In Organization of Memory (E. Tulving and W. Donaldson, Eds.), pp. 381-403. Academic Press, New York.

Ugurbil, K., Garwood, M., Ellermann, J ., Hendrich, K., Hinke, R., Hu, X., Kim, S.-G., Menon, R., Merkle, H., Ogawa, S., and Salmi, R. 1993. I maging at high magnetic fields: I nitial experiences at 4T. Magn. Reson. Q. 9: 259.

Vogeley, K., Kurthen, M., Falkai, P., and Maier, W. 1999. Essential functions of the human self model are implemented in the prefrontal cortex. Consciousness Cognit. 8: 343-363.

Worsley, K., and Friston, K. 1995. Analysis of fMRI time-series revisited-Again. Neurol mage 2: 173-181.

Zarahn, E., Aguirre, G. K., and d'Esposito, M. 1997. Empirical analyses of BOLD fMRI statistics. I. Spatially unsmoothed data collected under null-hypothesis conditions. Neurol mage 5: 179-197. 